Mitteilungen aus der pharmazentischen Abteilung des Eidgenössischen Polytechnikums in Zürich

\title{
Beiträge zur pharmazeutischen und chemischen Kenntnis der Cubeben und der als Verfälschung derselben beobachteten Piperaceenfrïchte.
}

\author{
Von Karl Peinemann.
}

(Eingegangen den 18. Dezember 1895.)

Der Gebrauch der Cubeben als Heilmittel schien in nenerer Zeit ein immer beschränkterer zu werden, es sah fast aus, als sollten dieselben das Schicksal so vieler anderer Drogen teilen, als sollten sie allmählich in der Rumpelkammer obsoleter Heilmittel ein bescheidenes Plätzchen angewiesen erhalten. Ganz unerwarteter Weise aber stieg der Verbrauch der Cubeben anfangs der achtziger Jahre bedentend, so bedeutend, dals die Kulturen nicht im Stande waren, den sich von Jahr zu Jahr mehrenden Bedarf zu decken. Die Preise erreichten eine erstaunliche Höhe und dieses ist wohl die Ursache, dals seit 1885 versucht wird, zahlreiche Surrogate auf den Markt zu bringen, die niit der echten Cubebe wenig oder gar nichts zu thun haben. Die grofse Zahl der Verfälschungen liels es wünschenswert erscheinen, dieselben übersichtlich zusammenzufassen, eine genaue systematische Untersuchung derselben vorzunehmen und zu zeigen, was gegenwärtig alles unter der Bezeichnung "Cubebe" vorkommt.

Auch dio Praxis wird aus einer solchen Arbeit vielleicht dadurch einen Nutzen ziehen, dafs ein in mancher Hinsicht sicher wertvolles Arzneimittel davor bewahrt wird, immer mehr in Miskredit zu kowmen, wie es der Fall sein muls, wenn der Gebrauch sich einbürgert, an Stelle der echten Droge Surrogate auf den Markt zu bringen, deren Heilwert im günstigsten Falle ein zweifelhafter ist. Es ist dieses der Zweck vorliegender Arbeit.

Während der Ausführung derselben gelangte ich zufällig in den Besitz einer grölseren Menge einer falschen Cubebe, wodurch ich in den Stand gesetzt wurde, dieselbe in chemischer Hinsicht einer ausfübrlicheren Untersuchung zu unterziehen, was mir bei den 
ubrigen Sorten, von welchen mir immer nur sehr geringe Mengen zu Gebote standen, leider nicht möglich war.

Auch die Ergebnisse dieser Untersuchung bilden den Inhalt der folgenden Blätter.

Die sehr zahlreichen Cubebensorten, es waren derer über sechzig, erhielt ich durch die grolse Freundlichkeit der Herren Professor Dr. B e ckurt s-Braunschweig, B r ü ckner-L a m p eBerlin, Ca e sar und Loretz-Halle, E. M. Hol mes-London, Professor Dr. S chä r-Stralsburg, B. Si e g fried-Zofingen, Dr. Tr e u b, Direktor des botanigchen Gartens zu Buitenzorg, Professor Dr. T s ch i r ch-Bern und Hofrat Professor Dr. Vogl-Wien. Es ist mir eine angenehme Pflicht, allen diesen Herren für die aufserordentlich bereitwillige Zusendung von Untersuchungsmaterial, sowie für freundlichst erteilte Auskünfte auch an dieser Stelle meinen verbindlichsten Dank zu sagen.

\section{A. Historischer Teil.}

Geschichteder Cubeben.

Die Cubeben lassen sich nicht früher als in der arabischen Medizin des Mittelalters nachweisen, wo sie den Namen Ka b ábo h . aus dem dann Cubebe entstanden ist, führen. Es ist dieses verhältnismälsig sehr späte Auftauchen der Cubeben einigermafsen auffallend, wenn wir in Betracht ziehen, dafs die verwandte Droge, der Pfeffer, weit früher bekannt wurde. Diesen finden wir bereits sicher erwähnt bei Theophrast, dem Schüler des Aristoteles und Plato und können wir mit ziemlicher Bestimmtheit annehmen, dals derselbe im A bendlande durch den Feldzug Alexanders nach Indien bekannt wurde.

I b n S in a, um 1006, giebt weben vielen anderen von ihm aufgezählten Eigenschaften des Kabábeh an, dals es ein gutes harntreibendes, Blasengries und Blasensteine vertreibendes Mittel sei A uch soll nach demselben Verfasser der Speichel, nach dem Kauen der Früchte, die geschlechtliche Thätigkeit erhöhen. M a s udi ${ }^{1}$ ) (10. Jahrh.) weifs, dafs sie aus Java stammen, ebenso Marco $\mathrm{Polo}$ ) (13. Jahrh.).

Die Pflanze selbst hat man erst viel später kennen gelernt. A l bertug $\mathrm{Magnu}{ }^{3}$ ) (12. Jahrh.) nennt sie einen niedrigen Baum

1) Flückiger, Pharmakognosie S. 927.

2) Ebenda S. 927 .

3) Ernst Meyer; Albertus Magnus, 2. Beitrag z. ernevert. Kenntnis d. bot. Leistungen. Linnaea XI, 1837. S. 565 . 
mit schmalen Blättern; gleicher Ansicht ist K o n r a d Mege n ber g 4), noch $\mathrm{Tab}$ arnaemontanus ) (Ende des 16. Jahrh.) vergleicht sie mit einem Apfelbaume, ebenso $\mathrm{S}$ chroeder 6 ).

Pierre Pomot 7) (17. Jahrh.) weifs, dals sie eine Schlingpflanze ist, auch Clusi us 8 ) erwähnt sì berejts als solche. Wissenschaftlich beschrieben wurde die Pflanze durch Linné, filius 1781.

Offenbar gelangte die Droge schon frühzeitig nach Europa; den oben erwähriten Schriftstellern sei noch hinzugefügt, die Heilige Hil d e gar d ${ }^{9}$ (12. Jahrh.). welche Cubebo anführt und ihm eine auffallend beruhigende Wirkung zuschreibt. Man könnte aber sagen, dafs diese Schrifteteller (Hildegard, Albertus Magnus, Megenberg) ihre Angaben anderen Autoren eninahmen, wir wissen jedoch, dafs Cubeben im Jahre 1153 in Aden Eingangazoll bezahlten auf dem Wege nach Europa ${ }^{10}$ ), sie waren dahin wohl auf dem Seewege gelangt. Im 14. Jahrh. treffer wir sie auf dem Landwege, der von Indien dureh Asien führte, in Tauris ${ }^{12}$ ), schon im 13. Jahrh. sind sie in London, Frankreich, Deutschland und Spanien auf dem Markte nachzuweisen. ${ }^{13}$ ) Offenbar war der soeben genannte Lundweg über Tauris und auch uber Bagdad ein sehr beliebter und pflegte man besonders kostbaro Aromata aus Indien auf dem sehr viel längeren und kostspieligeren Landwege auszuführen, weil man annahm, dafs sie auf dem kürzeren und billigeren Seewege (durch das Seewasser?) litten.

Vorzugsweise wurden die Cubelen jedenfalls als Gewürz verwendet, es ist aber falsch, zu sagen, dals ihre arzneiliche Ferwendung in Europa nicht über den Anfang dieses Jahrhunderts hinausreiche. (Braemer, Dymock, Dewèwre) Allerdings ist es richtig, dals ihre Verwendang als Arzneimittel fast aufgehört batte, und dals die englischen Militärärzte erst wieder die Aufmerksamkeit auf diese Droge lenkten, nachdem sie dieselbe bei ihren Hindudienern in Gebrauch gesehen hatten. Eine der ersten Arzneivorschriften, in welchex Cubeben wieder auftauchten, dürfte $\mathrm{Pi}$ isquins "Potio autigonorrhoica“ ron 1818 søin, welche ein wässeriger oder weiniger Auszug der Früchte war.13) Aus den zahlreiclien Beweisen für die frühzeitige medizinische Verwendung der Cubeben, auch in Europa, seien einge hier erwähnt:

1861. S. 326 .
4) Konr

5) Tabernaemontanus, Kräuterbuch, H. Bauhin 1731. II. S. 1331.

- Schroeder, Pharmac. nedico-chymica 1669. S. 3.

7) Pierre Pomet, Histoire générale des drogues 1694.

8) Garcia ab Orta, ed. Clusius 1601. I. S. 184.

9) Heilige Hildegard, Migne's Auegabe 1147.

10) Edrî̀î, Géographie, traduite par Amadée Tambert S. 31.

11) Heyd, Levantehandel im Mittelalter II., 83.

18) Flückiger, Dok. z. Geschichte d. Pharm. Nr. 6.

13) Pharmak. univers. Weimar 1832. I. S. 614. 
So wendete die $\mathrm{Schule}$ von $\mathrm{S}$ slerno dieselben im 12 . und

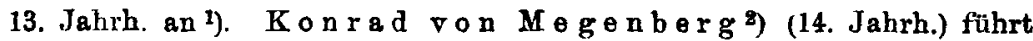
medizinische Verwendungen an. Ta bern a $\operatorname{con}$ o $\mathrm{tan}$ a $\mathbf{s}^{3}$ ) erwähnt ihren Gebrauch gegen eine ganze Reihe ron Krankheiten. Seb iz ${ }^{4}$ ) (1650) sagt: Cubobis officiris magis quam culinis inserviunt. S c hr 8 d $\theta \mathbf{r}^{5}$ ) kennt "Confectio cubebarum cum saccharo“, also mit Zucker überzogene Cubeben, ein Cubeben en thaltendes Theegemisch und das atherische Oel, er sagt, dafs man sie selten gebrauche. Auch in vielen Pharmakopöen des vorigen Jahrhunderts wird der Cubeben Erwähnung gethan. So finden wir sie inder Pharmacopoea universalis J.R. Spielmann (1783), in der $P$ harmacopoea Wirtenbergica (1771)

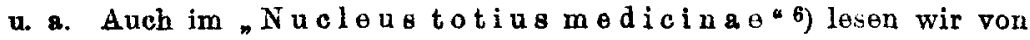
der mannigfachen Zubereitung und Anwendung der Cubeben als Heilmittel. Einen hochinteressanten Beweis für die auch früher stattgehabte Verwendung in der Medizin finden wir schlitslich in einer alten Dissertation über Cubeben vom Jahre 1705 7). Auch hier wird hervorgehoben, dals „cubebae sunt aroma medicamentosum, minus alimentosum*. In sehr ausführicher Weise ergeht sich der Verfasser in der Beschreibung der verschiedensten Anwendungen in der Medizin und fuhrt zum Schluss eine ganze Reihe von Heilmittelmischungen an, in welchen der Cubebe die wesentlichste Rolle zukommt.

Von Verfälschungen der Droge ist in dieser alten Zeit wenig diu Rede, immerhin scheint sie nicht völlig davon verschont geblieben zu sein. So berichten Albertus Magnus ${ }^{8}$ ) und Megenberg ${ }^{9}$, dafs man sie mit Wachholderbeeran verfalsche, wie das Gleiche auch vom Pfeffer orwähnt wird.

Bei dem Bestreben der mittelalterlichen Medizin, möglichst die von den Alten gebrauchten Heilmittel zu verwenden und also die zur Verfügung stehenden mit den von den Alten genannten zu identificieren, kann es nicht Wunder nehmen, dals man sich auch bemühte, die Cubeben mit einer im Altertum bekannten Droge in Uebereinstimmung zu bringen. So fal'ste für die Cubeben der Gedanke Wurzel, dieselben seien mit uiner, in alter Zeit of genanuten Droge, dem Carpesium, identisch und noch im 16. Jahrh. finden wir Cubeben a]s Fructus Carpesiorum genanut. Carpesium wird von den alten Schriftetellern bald als eine Frucht, bald als ein Holz bezeichnet, als

1) Flückiger, Pharmakognosie S. 927.

2) Konr. v. Megenberg, 1. c.

3) Tabernaemontanus. 1. c.

๑) Flückiger, 1. c. S. 928 .

5) S:hröder, l. o.

6) Nucleus tot. med. v. Arth. Konr. Ernstingium. 1770. S. 355.

7 Dissert. inaugur. med, de cubebis v. H. Fr. Teichmeyer, Jena 1705.

8) Albert. Magnus, 1. c.

9) Konrad $\vee$. Megenberg, 1. c. 
letzteres scheint es in gewissen Beziehungen zam Zimt zu stehen. Es wird gegenwärtig augenommen, dafs man unter den als Carpesium bezeichneten Früchten diejenigen einer Xanthoxyleenart zu verstehen habe.

Zu der Annahme, dafs Carpesium mit Cubeben identisch seien, dürfte wohl in erster Linie die ähnliche Wirkung beider Veranlassung gegeben haben. Schon Pa u u $\mathbf{s}$ A egineta ${ }^{1}$ ) erzäblt von der Wirkang des Carpesiums auf den Harnapparat und schreibt demselben Heilwirkungen $\mathrm{zu}$, welche sich mit den für Cubeben von älteren Autoren angegebenen vollständig decken. Möglich ist auch, dafs dio als Carpesium bezeichnete Droge sls Verfälschung oder Substitution der Cubebe aufgetreten ist, vielleicht mit solcher Beharrlichkeit, dals man schliefslich zwischen Carpesium und Oubeben nicht mehr zu unterscheiden wufste. Dieser Grund für die Uebertragung der Bezeichnung Carpesium auf die Cubebe erscheint nicht mehr so gewagt, wie auf den ersten Blick, wenn man erwägt, dafs noch in allerneuester Zeit Xanthoxyleenfrüchte, wahrscheinlich die von Evodia rutaecarpa Benth., als Cubeben vorgekommen sind und wenn man bedenkt, dafs dem berahmten Bruder der Cabebe, dem Pfeffer, etwas ähnliches passierte. Im 16. Jahrh. wird ihm verschiedentlich eine Frucht substituiert, die den Namen Fagara führt und welche von Xanthoxylum Budrunga DC. und Xanthoxyl. Rhetsa DC. geliefert wurde.

Unter dem Namen Faghireh beschreibt bereits I b $\mathrm{S}$ ina (1006) Xanthoxyleenfrüchte.

Noch heutigen Tages werden solche Früchte als scharfschmeckendes Gewürz verwendet, so bei den Chinesen, welche eine Xanthoxyleenaxt "Hwa-tseau" oder Pfefferblume nennen, auch in Japan sind die Früchte von Xanthox. piperitum in Gebrauch. ${ }^{2}$ ) Eine ganze Anzahl von Früchton aus der Familie der Xanthoxyleen führt $R$ os en tha ${ }^{3}$ ) als scharfschmeckend an, so Xanth. ternatum Sw., Xanth. alatum Roxb., Xanth. senegal. DC., Xanth, piperit. DO., deren Samen, baccae Fagarae seu Piper japonicum, wie schon erwähnt, als Gewürz dienen. Die reifen Samen von Xanth. Rhetsa DC. sollen fast die Schärfe des schwarzen Pfeffers besitzen.

Wir sehen also, dafs Xanthoxyleenfrüchte zur Verfälschung resp. Substitution des Pfeffers gedient haben, andererseits lafst sich aber nachweisen, dals die Beziehungen der Fagara zu den Cubeben entschieden grölsere sind. So finden wir bei Háji-Zein und Attár, welche in Jahre 1368 schrieben, orwähnt, dals die Perser Fagara unter dem Namen „Kabábe i kas hádeh" (Cubeben mit offenem Mund) kannten, so dafs sie hier direkt unter dem alten Namen

1) Paulus Aegineta, Basilea 1546 I. VII p. 586.

2) Dymock, Pharmucographia Indica, Vol. I. S. 256.

3) Synopsis plantaram diaphoricarum, S. 874 u. 875. 
der Cubeben genannt wird. In der That ist diese Bezeichnung gar nicht ungaschickt gewählt, wenn man die braune Farbo der Fagarafrüchte und ihre im Reifezustande auseinander klaffende Fruchtschale in Betracht zieht. Auch C. B a u h i $\mathbf{~}^{\mathbf{l}}$ ) batont ausdrücklich dio Ashnlichkeit der als Fagara bezeichneten Drogo mit Cubo'sən, wobai wohl kaum an die echten gedacht werden kann.

Fassen wir das hier Gesagte zusammen, so dürfte daraus hervorgehen, erstens, dals wir unter Carpesium Xanthoxyleenfrüchte zu verstehen haben und dals diese zweitens mit der als Fagara bezeichneten Substitution des Pfeffers zum mindesten nahe verwandt wenn nicht identisch sind, ein nicht uninteressanter Beweis, in ein wie hohes Alter Substitutionen von Drogen, die noch heute vorkommen, hinaufreichen können.

Geschichteder Bestandteile.

Nit der Analyse der Oubeben haben sich verschiedene Forscher beschäftigt, so von älteren: $\nabla a q_{q u} \operatorname{lin}^{2}$ ), Trommsdor $f^{8}$ ), Monheim4, u. a. m. Neuere Arbeiten haben geliefert: Bernatzik ${ }^{5}$ ), Schmidt ${ }^{6}$ ) and $S c^{-h u l z} \theta^{7}$ ). Erat durch diese letzteren Arbeiten und namentlich durch die sehr ausführliche von $\mathrm{S} \mathrm{chmidt}$ haben wir Kenntnis von sämtlichen in den Cubeben enthaltenen Stoffen bekommen, von welchen als wichtigste zu nennen sind: Aetherisches Oel, fettes Oel, Cubebin, Cubebensäure und indifferentes Harz. Die bekannte therapeutische Wirkung der Droge kommt nur den letzten beiden Bestandteilen zu.

Das a therische $\mathrm{O}_{\theta 1}$ war bereits verhaltaismäfsig früh bekannt, nach FI $\mathfrak{u}_{\mathrm{c}} \mathrm{k} \mathrm{g} \mathrm{er}^{8}$ ) wird dasselbe in dem Invent ar der Ratsapotheke zu Braunschweig 1609 aufgeführt. Wir kön nen hinzufügen, dufs es schon im Jahre 1580 in einer Beschreibung der Frankfurter Messe erwähnt wird9). DaLs man so verhältnismäfsig frübzeitig die Cubeben der Destillation mit Wasserdämpfen unterwarf, kann nicht Wunder nehmen, wenn man bedenkt, dafs das ätherische $O e l$ des verwandten Pfeffers schon in der ersten Hälfte des 16. Jahrhu nderts von Valerius Cordus dargestellt wurde.

1) Tabernaemontanus, 1. c.

2) Buchner's Repert. d. Pharm. Bd. XI. S. 339.

3) Trommsdorff's Journ. d. Pharm. Bd. XX. S. 69.

4) Buchner's Repert. d. Pharm. Bd. 44. S. 199.

5) N. Repert. d. Pharm. Bd. 14. S. 98.

6) Archiv d. Pharm. 1870 S. 1.

7) Archiv d. Pharm. 1873 S. 393.

8) Flückiger, Pharmakognosie.

9) Fläckiger, Dokumente z. Geschichte d. Pharm.

Arch. d. Pharm. CCXXXI V. Bds. 3. Heft. 
Das Oel ist in den Cubeben in sehr wechselnden Mengen enthalten, die Ausbeute kann nach $\mathrm{S}$ chim mel \& $\mathrm{Co.1}^{1}$ ) zwischen 7 und 16 pCt. schwanken. Auch die Farbe desselben ist nicht immer dieselbe, sie wird beeinflulst durch ein in den letzten Fraktionen enthaltenes blaues $\mathrm{Oel}$, welches je nach Alter oder vielleicht auch nach dem verschiedenen Reifezustande der Früchte in gröfiserer oder geringerer Menge vorhanden sein kann. Das zuerst übergehende Oel is t farblos, dtinnflüssig und stark lichtbrechend, während die später nubergetriebenen Anteile dicktlüssiger, stark gefürbt und weniger stark lichtbrechend sind. Schmidt unterscheidet daher zwischen einem leichten und einem achweren Oele.

Mit der Untersuchuig des Cubebenoles haben eich aufser den genannten Forschern eingehender beschäftigt: $O \mathrm{~g} l$ i a 1 o r o ${ }^{2}$ ), welcher nachwies, dals dasselbe aus einer geringen Menge eines zwischen 158-1630 siedenden linksdrehenden Terpenes, einem starker linksdrehenden, bei $264^{\circ}$ übergehenden Kohlenwasserstoff von der Formel $\mathrm{C}_{15} \mathrm{H}_{24}$, welcher wit $\mathrm{H} \mathrm{Cl}$ eine krystallinische Verbindung eingeht und ans einem wahrscheinlich ebenfalls obiger Formel entsprechenden Kohlenwasserstoff, der sich nicht mit $\mathrm{H} \mathrm{Cl}$ vereinigen lasist, besteht. W a $11 \mathrm{ach}^{3}$ ) bestätigt die schon fruher gemachte Beobachtung, dals das Cubebenöl auch unter $200^{\circ}$ siedende Anteile enthält. Er stellte aus diesen die Chlorwasserstoffverbindung $\mathrm{C}_{10} \mathrm{H}_{18}, 2 \mathrm{HCl}$ dar, wies also die Anwesenheit von Dipenten in dem Oele nach. Wallach gab dem in der bei 250-2700 tibergehenden Hauptmenge enthaltenen Sesquiterpene $\mathrm{C}_{15} \mathrm{H}_{26}$, ein Kohlenwasserstoff, welcher sich weit verbreitet im Pflanzenreiche vorfindet, den Namen $\mathrm{C}$ a dinen. Wir können jetzt also sagen, das Cubebenöl besteht im Wesentlichen aus Dipenten und Cadinen.

Aus dem Oele älterer Cububen scheidet sich bei langerer Abkahlung der $\mathrm{Cub}$ eben a mpher aus, ein in geruchlosen, rhombischen Oktaëdern krystallisierender Körper von der Zusammensetzung $\mathrm{C}_{15} \mathrm{H}_{24}, \mathrm{H}_{2} \mathrm{O}$, welcher zuerst von $\mathrm{Blanchet}$ und $\mathrm{Sel} 1^{4}$ ) naber studiert wurde.

Das Cubobin, etwa gleichzeitig von $\mathrm{Ca}$ a o ola und $\mathrm{M}$ onhoim entdeckt, wurde zuerst von Soubeiran und $C_{a p i t a i n}$ ) krystallisiert erhalten. Es findet sich nach $\mathrm{S}$ o h m i d t zu etwa 2,5 Proz. in den Cubeben. Mit konz. Schwefelsäure giebt es die prachtvoll purpurviolotte Färbung, welche als charakteristische Realrtion für echte Cubeben anzusehen ist, auch giebt es eine Reihe von Reaktionen,

1) Schimmel \& Co., Berichte.

2) Berichte 1875, S. 1357.

3) Annalen d. Chem. 238, s. 78.

4 Annalen d. Chem. 6, S. 294.

5) Journ. Pharm. (2) 25, S. 355. 
welche leicht mit denjenigen gewisser Alkaloide verwechselt werden Iónnen.1) Mit der Konstitution, Darstellung verschiedener Derivate etc. beschaftigte sich $W_{\theta i d e 1.2}$ ) Derselbe stellte durch Einleiten von $\mathrm{N}_{2} \mathrm{O}_{3}$ in atherische Cubebinlösung das Nitrccubebin, $\mathrm{C}_{10} \mathrm{H}_{9}\left(\mathrm{NO}_{2}\right) \mathrm{O}_{3}$ dar; durch Einwirkung von Salpetersäure auf Cubebin erhielt er Oxalsăure und Pikrinsäure.

Ich will hier schon bemerken, dals ich beim Behandeln des Cubebins mit Salpetersäure zu einem wesentlich anderen Resultate kam, ich erhielt nur als vollstandig nebensächliche Einwirkungsprodukte Oxalsäure und Pikrinsäure in sehr geringen Mengen, der Hauptsache nach aber einen Nitrokörper in wobl auegebildeten Krystallen, von welchem später die Rede sein wird.

Durch Einwirkung von Brom auf in Chloroform gelostes Cubebin gelangte $\mathrm{W}$ eidel zu dem Körper $\mathrm{O}_{10} \mathrm{H}_{7} \mathrm{Br}_{3} \mathrm{O}_{2}$, welcher wohl unrichtig als Tribromeubebin in manchen Lehrbüchern bezeichnet wird, da er vielmehr eir durch Wasseraustritt entstandenes Bromsubstitutionsprodukt darstellt. Kaliumbydroxyd mit Cubebin geschmolzen, lieferte genanntem Verfasser: Kohlensäure, Essigeäure und Protocatechusäure als Zersetzungaprodukte.

P omeranzs) beschäftigte sich mit Erforschung der Konstitution des Cubebins, es gelang inm unter den Oxydationsprodukten desselben Piperonylsäure nachzuweisen, entstanden durch Oxydation von Cubebin mittels Kaliumpermanganats in alkalischer Lösung. Er konnte ferner eine Hydroxylgruppe im Cubebin nachweisen, indem er dasselbe durch Einwirkung von Benzoylchlorid esterifizierte. Eine weitere Bestätigung der Alkoholnatur des Cubebins lieferte inm der Versuch, dieses uach der Liebermann'schen Methode zu acetylieren, wodurch er allerdings nicht das zu erwartende Acety derivat, sondern den Cubebinaether erhielt. P o meranz gelangt so schlielslich zur Aufstellung einer Formel, die allen genannten Reaktionen Rechnung trägt, dennoch aber nicht die richtige sein dürfte, da Cubebin, als optisch aktiver Körper ein asymmetrisches Kohlenstoffatom enthalten mufs, dieses aber in der von Pom $\theta \mathrm{ranz}$ aufgestellten Formel nicht enthalten ist.

A. Angeli und P. Mol $\left.e^{1}\right)$ studieren die Frage, ob es nicht möglich sei, Cubebin $\left(\mathrm{C}_{10} \mathrm{H}_{10} \mathrm{O}_{8}\right)_{2}$ in Diisosaffrol $\left(\mathrm{C}_{10} \mathrm{H}_{10} \mathrm{O}_{2}\right)_{2}$ uberzufübren und damit aus der Konstitution des letzteren Schlufsfolgerungen auf diejenige des ersteren zu ziehen. Die Verfasser sind mit ihren Arbeiten zur Zeit noch nicht zu einem Abschluls gelangt. Aus ihren vorläufigen Mitteilungen ist vielleicht erwähnenswert, dafs sie bei

1) Schaer, Reaktionen d. Cubebins, Archiv d. Pharm. 1887.

3) Wiener academ. Sitzungsberichte (2) 74, \$. 377 .

3) Monatshefte f. Chem. 1887, S. 466 und 1888, S. 323,

4) Gazzetta chimic. ital. 1894, S. 127. 
Einwirkung von Brom auf Cubebin unter genau den von W e i del gegebenen Bedingungen zu einem Bromprodukte gelangt sind, welches mit dem von $W$ e idel dargestellten nicht identisch ist. Während der von letzterem dargestellte Körpar der Formel $\mathrm{C}_{10} \mathrm{H}_{7} \mathrm{Br}_{2} \mathrm{O}_{2}$ entspricht, kommt dem von $\mathrm{A} \mathrm{ng}$ eli und $\mathrm{Mol} \theta$ erhaltenen die Formel $\mathrm{C}_{10} \mathrm{H}_{8} \mathrm{Br}_{2} \mathrm{O}_{2} \mathrm{zr}$.

Eykman $n^{1}$ ) stellte nach der kryoskopischen Methode für Cubebin die Molekularformel $\mathrm{C}_{20} \mathrm{H}_{20} \mathrm{O}_{6}$ fest.

Die $\mathrm{Cubeben} B \ddot{a} u r \theta$, eine Harzsäure, für welche $S \mathrm{ch}$ id die Formel $\mathrm{C}_{13} \mathrm{H}_{14} \mathrm{O}_{7}$, Sch ulze aber $\mathrm{C}_{28} \mathrm{H}_{30} \mathrm{O}_{7}, \mathrm{H}_{2} \mathrm{O}$ aufstellte, wurde schon von Monheim beobachtet, von Bernatzik aber erst genauer beschrieben, von $\mathrm{Schmidt}$ und auch von $\mathrm{Schulz} \theta$ ausführlicher untersucht. Sie bildet eine weilse, harzartige, zu otwa $1,7 \%$ in den Cubeben enthaltene, nicht krystallisationsfähige Masse, welche nach $S$ ohm id bei $56^{\circ}$, nach $S \circ$ h u $1 \mathrm{ze}$ bei $45^{\circ}$ schmilzt und sich an der Luft allmählich bräunt. Ihre $\mathbf{R}$ eaktion ist nur schwach sauer. Mit Ausnahme des Natriumsalzes, dessen Darstellung in Krystallen $\mathrm{S} \mathrm{chulze}$ gelang, sind sämmtliche Salze nur in amorphem Zustande erhalten worden.

Das fette $\mathrm{O}_{\text {el }}$ der Cubeben bildet eine ziemalich dickflüssige, bei $n^{0}$ salbenartig erstarrende, in kaltem Alkohol langsam, aber vollständig sich auflösende Masse von tief olivengrüner Farbe.

Das ind ifferente $\mathrm{H}$ arz, welches neben der Cubebensäure den wirksamen Bestandteil der Cubeben ausmacht, bildet eine gelbbraune Masse, welche sich leicht in Alkohol, schwer in Chloroform, Aether und Schwefelkohlenstoff auflöst. Nach Schmidt entspricht seine Zusammensetzung der empirischen Formel $\mathrm{C}_{13} \mathrm{H}_{14} \mathrm{O}_{5}$.

Handelsgeschichtlichesund Uebersicht der beobuchteten Verfälschungen.

Der Handelswert der Drogen ist von jeher ein schwankender gewesen, standen dieselben hoch im Preise, so reizte der zu erzielende Gewinn die Sammler, Pflanzer und Exporteure za grölseren Zufuhren, eanken sie dann in Folge des Ansammelns von Vorräten, welche die voraussichtliche Nachfrage auf Jahre hinalas zu ducken im Stande waren, unter eine gewisse Norm, so fanden genanate Kreise, d als sie ihre Zeit mit nutzbringenderen Beschäftigungen, als mit dem Einsammeln oder Kultivieren von Arzneipflanzen verbringen könnten, die Zufuhren blieben aus und der Preis ging naturgemills wieder in die Höhe, worauf dasselbe Spiel von vorne anfing. Solche regelmälsigen Schwankungon sind überall zu bemerken und leicht zu konstatieren, daneben treteu aber bisweilen ganz plötaliche abnorme auf, deren Ursachen durchaus nicht immer sofort in die Augen springen, sodals

1) Berichte, 23, S. 856 . 
die Nachforschung nach diesen oftmals interessant ist. Eine solche a bnorme pl ötzliche Schwankung Jäfst sich bei den Cubeben konstatieren.

Einen ungewöhnlich niedrigen Wert hatten Cubeben Ende der sechz jger und Anfang der siebziger Jahre, wo derselbe kaum ein Drittel des früheren erreichte. Im Jahre 1873 zeigte sich eine plötzliche, doch nur vorübergehende Besserung. Es wurde behauptet, dals in Java, wegen nicht mehr lohnend gewesener Kultur, die Stauden herausgerissen und durch Pfefferanplanzungen ersetzt seien. Die Spekulation bemächtigte sich der Droge, es wurde in Holland alles, was nur zu haben war, aufgekauft und die Preise um mehr als 50 Proz. in die Höhe getrieben. Die Ursache dieser plötzlichen Erhöhung konnte-sich nicht mit Sicherbeit ermitteln lassen, nach der einen Angabe soll der Krieg Hollands mit Atschin, durch welchen das Ausbleiben fernerer Zufuhren erwartet wurde, der Grund gewesen sein, nach anderer Lesart soll die Droge damals von Frankreich sehr begehrt worden sein, um zum Vermischen, resp. Verfälschen des Pfeffers zu dienen. Wie dem aber auch sei, Thatsache ist, dafs der Wert der Cubeben in sehr kurzer Zeit wieder ąuf den früheren niedrigen Stand angelangt war. Bis zum Jahre 1880 war der Artikel nur geringen Preisschwankungen unterworfen, zu welcher Zeit dann aber ein plotzlicher Umschwung eintrat. Amerika, welches tubrigens schon immer eine gunz besondere Liebhaberei für Cubeben gehabt hatte, hatte auf einmal dieselben ala hervorragendes Universalmittel erkannt und empfahl besonders ihre Anwendung gegen katarrhalische Leiden und ihren Gebrauch gegen Asth ma in Form von Cigaretten. Von Amerika aus wurde nun alles was an Cubeben zu haben war, aufgekauft, so dafs der Preis derselben eine noch nie dag ewesene Höhe erreichte. Fs möge gestattet sein hier die interessanten Preisschwankungen für die letzten Jahre anzuführen, interessant, weil meines Wissens noch nie eine Droge ähnliche durchzumachen gehabt hat:

Das halbe Kilogramm bester Ware wurde, auf dem Weltmarkte gekauft, in Cents holländischer Wärung bezahlt mit:

$\begin{array}{llllllll}1875 & 1876 & 1878 & 1879 & 1880 & 1881 & 1882 & 1883\end{array}$

11 Cts. 14 Cts. 21 Cts. 33 Cts. 55 Cts. 85 Cts. 125 Ots. 240 Cts. $\begin{array}{llllll}1884 & 1887 & 1888 & 1889 & 1890 & 1891\end{array}$

245 Cts. $\quad 300$ Cts. 325 Cts. $340 \mathrm{Cts}$. $300 \mathrm{Cts} . \quad 130 \mathrm{Cts}$.

$1892 \quad 1893 \quad 1894$

65 Ots. $\quad 45$ Cts. 25 Cts.

Leider war es mir nicht möglich, aus der Litteratur auch die Ausfuhren von Java festzustellen, die Vorräte der Hauptstapelplatze London und Amsterdam gaben ebenfalls keine Anhaltungspunkte, da grofse Posten direkt nach Amerika exportiert wurden. Ich mufs mich daher darauf beschränken, den Export Java's während der Jahre 1889-84, für welche Ziffern vorliegen, hier anzuführen. Derselbe giebt 
immerhin ein Bild, in welch grofsem Mafsstabe die Ausfuhr durch die starke Preissteigerung beeinflufst worden ist.

Die Ausfuhr von Java betrug in Piculs (1 Picul $=601 / \mathrm{a} \mathrm{kg})$ vom 1. Juli 1889 bis 30 . Juni $1890=1353$ Piculs.

$\begin{array}{lllllllll} & 1 . & & 1890 & & 30 . & & 1891=1378 & \end{array}$

Bei der grofsen Nachfrage, welche um 1880 begann, waren dio vorhandenen grofsen Vorrate, welche in Amsterdam undLondon lagerten, bald zusammen geschmolzen, und da auch Java und Ostindien, wo die Cubebenanpflanzungen anderen Kulturen hatten weichen müssen, nicht in einer der Nachfrage entsprechenden Weise für Zufuhren sorgen konnten, so ist der riesige Aufstieg im Preise wohl erklärlich. Wie bei fast allen Drogen, wenn sie recht hoch im Preise stehen, der Fälscher sein Handwerk beginnt und minderwertige, verdorbene, oder gar vollständig von der eigentlichen verschiedene Waare an den Mann zu bringen sucht, so war dieses Schicksal den Cubeben ebenfalls nicht vorenthalten. Bis zum Jahre 1885 hatte man von Verfälschungen der Cubeben wohl hier und da ausnahmsweise etwas gehört, doch waren eigentliche absichtliche Unterschiebungen anderer Frächte wenig beobachtet, jedenfalls in gar keinem annähernden Verhältnisse zu der in diesem Jahre beginnenden Ueberflutung mit allen möglichen Surrogaten stehend.

Als Verfälschungen resp. Verwechslungen werden vor 1885 vor nehmlich erwähnt') (vergl. auch Historisches): Fructus Rhanni cathart, Frust. Amomi, Piper ribesioides Wall., Cubaba Noesii Miq., Piper nigrum, Piper caninum Viq., Piper anisat. Humb. u. Bonpl., Piper crassipes Korth., Piper Lowong BI. Es muls hier auf. tallen, dafs einige dieser Früchte gerade jetzt, wo doch Verfälschungen der Cubeben im grofien aufgetreten sind, nicht mehr als Substitution der letzteren angetroff der falschen Sorten naturgemäls ein weit eingehenderes und zuverlässigeres geworden ist. Es liegt daher die Wahrscheinlichkeit nahe, dafs die damalige richtige Bestimmung der Verfälschungen nicht über allen $\mathrm{Z}$ weifel erhaben ist,

Eine verschiedentlich erwähnte „Beisorte* mag ebenfalls hier ibren Platz finden, sie soll aus völlig ausgereifien Cubeben bestehen und ist daher unzulässig, da, wie bekannt, nur die unreifen Früchte die Handelsware bilden sollen. Schliefslich möge noch Piper borbonnense hier angeführt werden, der vielleicht hier und da auch als Verfälschung

1) Yharmakognosion von Schroff 1853, S. 114, Wiggers 1853, S. 141, Flückiger 1867, S. 614, Henkel 1867, S. 347, Wigand 1879, S. 286 , u. a. m. 


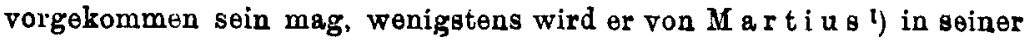
Pharmakognosie unter "Cubeben" erwähnt. M a rti u s sagt: „Cubeben von Bourbon sollen von der Gröfse eines Hirskornes sein", und in der That sind die mir vorliegłnden Früchte von Piper borbon. von auffallender Kleinheit.

Wie gesagt, alle diese verschiedenen Früchte waren wohl als Verfälschungen auch früher schon vorgekommen, dieselben hatten aber doch niemals eine beunruhigende Ausdehnung erreicht und hatten auch niemals ein übermäfsiges Interesse erregt. Ganz anders von 1885 an. Die von diesem Jahre an auftauchenden Surrogate bestehen im grofsen und ganzen allerdings auch nur aus den oben erwähnten Früchten, doch die grolse Masse der nun al den Markt gebrachten Verfälschungen ist es, was die Aufnerksamkeit erregen mulste.

Naturgemäls finden wir in der englischen Litteratur die ersohöpfendsten und weitıus zahlreichsten Angaben hierüber. Es wärde zu weit fuhren, wollte ich an dies :r Stelle eine genaxe Bescireibunz der aufgetretenen Verfälschungen geben, ich begnüge mich dэshalb hier mit kurzen Litteraturangaben. So wird das Auftauchen falscher Cubeben, und zwar von Daphnidium Cubeba N. ab E. im Pharmaceutical Journal and Transactions ${ }^{2}$ ) erwähnt. W. Kirkby giebt ebendaselbst, Seite 653, eine genaue Beschreibung einer grölseren, hejleren Sorte, mit stärkerem Stiel und abweichendem Geruch. S. 909 erwähnt $\mathrm{H}$ ol $\mathrm{m} \theta \mathrm{s}$ falsche Cubeben, welche einen an Macis erinnern den Geruch und eiven bittereren Geschmack als die echten Frtichte besitzen. S. 1005 spricht Ed. Gravill von „spurious cubebs", ohne aber nähere Beschreibung zu geben. In derselben Zeitschrift ${ }^{3}$ ) wird uber Krankheitserscheinungen beim Gobrauche des Cubabenpulvers geklagt. Es zeigte sich bei näherer Untersuchung des Pulvers, dals dasselbe aus stark mit Daphnidiumfrüchten untermischter Droge hergistellt war, Ebendaselbst S. 197: Auftreten falscher Cubeben in New-York. S. 545: Auftreten solcher in England. In demselben Journal 4) werden Fruchte beschrieben, welche in Farbo und Gröfso den echten gleichen, keinen Stiel besitzen, mit einem fünfzähuig $\rightarrow n$ Kelch, zwei vertikalen Eindrücken und zwei reohtwinklis auf den. selben stehenden Erböhungen. Im gleichen Bande, S. 508: Es wurden zwischen offizinellen Cubeben Früchte von Evodia rutaecarpa Benth und vielleicht noch anderen Rutaceen gefunden. S. 621 wird eine Sendung beschrieben, die aus Rhamnusfrüchten, umreiten Früchten der sishwarzen $P$ feffers, $P$ fefferstielen, Blüten von Alpinia und einigen echten
1) Martius, Pharmakognosie 1832, S. 223.
2) Pharm. Journ. and Trans. Ser. III (15) S. 614.
3) Pharm. Journ. and Trans. Ser. III (15) S. 107.
4) Pharm. Journ. and Trans. Ser. III (17) S. 310. 
Cubeben bestand. Kir k b y ${ }^{1}$ ) bespricht eine Cubebe, welche im Pericarp Sklerose enthält. S. 481 werden hellere Frlachte, anscheinend unreife Cubeben erwähnt, auch $S .846$ werden unreife Früchte angeführt. In derselben Zeitschrift ${ }^{2}$ ) wird von Früchten berichtet, die grauer und stärker gerunzelt sind, als die echte Droge, und die mit konz. Schwefelsäure keine Cubebinreaktion geben. Ich kann hier nicht unterlassen, einige Worte binsichtlich der mehrfach als Verfälschung erwähnten unreifen Cubeben zu bringen. Es ist der Ausdruck „unreif" meiner Ansicht nach beiVerfälschungen vonCubeben nicht gerade am Platzt. Die Handelsware, die echten Cubeben, soll ja gerade in unreifem Zustande gesammelt werden und als unreife Früchte finden sich dieselben auch in allen Pharmakopoeen beschrieben. Uebrigens sind auch ganz junge, echte Cubeben stets ohne weiteres zu erkennen.

Der falschen Cubeben wird auch von $\mathrm{Geh}$ e u $\mathrm{d} \mathrm{Co}^{\mathrm{g}}$ ) Erwähnung getban, ebenso beschreibt $\nabla \circ g 1^{4}$ ) verschiedene ihm zu Gesicht gekommene Sorten unechter Frikchte.

Ich möchte nicht behaupten, dal's alle vorliegenden Verfälschungen absichtliche gewesen sind, bei dem Mangel an echten Cubeben und bei dem in Aussicht stehenden grofsen Gewinn, haben die Javaner, denen wohl keine grofsen botanischen und pharmakognostischen Kenntnisse zur Seite steken, in ihrem Uebereifer sicher oft alle nur irgerd erreichbaren Früchte, sofern dieselben nur einige A ehnlichkeit mit Cubeben hatten, in gutem Glauben als solche verkauft.

Bei manchen Surrogaten fiel die Erkennung für ein einigermafsen getbtes Auge nicht schwer, fremde Früchte, wie Tetranthera, Daphnidium, Rhamnus, waren ohne besondere Untersuchung leicht zu erkennen. Rhamnusfrüchte können meiner Meinung nach nicht einmal als eigentliche Verfälschung betrachtet werden, sondern sind mehr als Verunreinigung anzusehen. Sie kommen doch wohl eher aus Unachtsamkeit, als aus echlechter Absicht, beim Sammeln unter die guten Cubeben und sind in dew meisten Handelssorten letzterer zu finden. Vielleicht läfst sich ihr Vorkommen nicht unpassend mit der Beimengung vergleichen, welche gewisee Sorten von Fol. Sennae durch Arghelblätter in regelmalsiger Weise erfahren.

Etwas leichtere Verwechslung könnte schon stattfinden, bei den aus einer anderen Abteilung der Gattung Piper stammenden, ungestielten Pjperaceenfrüchten wohin z. B. schwarzer Pfeffer und die von

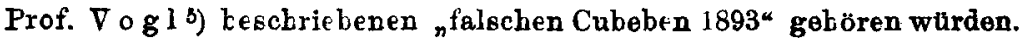
Grölsere Schwierigki iten bereiten die, wie die echte Cubebe, der gleichen

1) Pharm. Journ. and Trans. Ser. III (18) S. 269.

2) Pharm. Journ. and Trans. Ser. III (21) S. 614.

3) Gehe \& Co., Handelsberichte 1885 u. folg.

4) Pharm. Post 1894. S. 482. Vogl, uber Cubebensorten.

5) Vogl, l. c. S. 482 . 
Abteil ung Cubeba, Sch wanzpfeffer, angehörenden, mit sogenanntem Stiel versehenen Früchte. Ich sage „sogenannt“, da der Ausdruck „Stiel“ im morphologischen Sinne als unrichtig bezeichnet werden mufs. Die Früchte besitzen keinen Stiel (pedunculus), sind also wie andere Piperaceenfrüchte sitzend und haben nur einen stielartigen Fortsatz des Pericarps. Zur Unterscheidung der verschiedenen, zur Abteilung Cubeba gehörenden Früchte ist mikroskopische und chemische Prüfung erforderlich. Mikroskopische allein reicht meines Erachtens nicht aus, da Sorten mit völlig identischem Bau bei ihrer Behandlung mit mikrochemischen Reagentien wesentliche Unterschiede zeigen. So giebt es, um ein Beispiel anzuführen, drei Varietäten von Piper Cubeba, welche von den Eingeborenen Java's als Rjnoe katoentjar, $R$ badak, $R$. tjaroeloek bezeichnet werden und von welchen ich die ersten beiden durch Herrn Dr. Tre u b-Buitenzorg erhielt. Die bejden Sorten waren äufserlich durchaus ähnlich und liefsen auch mikroskopisch sich durch nichts von einander untersoheiden. Beim Behandeln der Schnitte mit konz. Schwefelsäure zeigte sich aber bei der einen Frucht (var. katoentjar) die intensive purpurviolette Färbung, also die charakteristische Reaktion fur Cubebin, während die andere (var. badak) bei gleicher Behandlung eine durchaus gelbe Farbe zeigte, ein Beweis, dafs letztere kein Cubebin enthält. Ich bemerke hierbei, dafs beide untersuchten Sorten sich in völlig gleichem Reifezustande befanden.

$\mathrm{Ob}$ dergleichen Unterschiede in botanischer Hinsicht als durchgreifend genug zu betrachten sind, um eine Trennung der Art Piper Cubeba als wünschenswert erscheinen zu lassen, wage ich nicht zu entscheiden. Im allgemeinen wird man sagen können, dals die Botaniker abgeneigt sind, solche Unterschiede systematisch zu verwenden, vielleicht mit Recht. Ich erinnere hier nur an den Mandelbaum, von welchem eine Varietät süfse, eine andere bittere, Amygdalin enthaltende Früchte hervorbringt. Trotzdem dieselben in chemischer Hinsicht sehr grofse Verschiedenheit zeigen, hat man doch nicht auf Grund derselben eine Trennung der Art für angezeigt gehalten.

Als ich mit dem mikroskopischen Teil dieser Arbeit bereits vollständig fertig war, wurde mir zufällig durch ein Referat im PharmJourn. and Trans. Kenntnis von der durch A. D $\theta \mathrm{w} \dot{\theta} \mathrm{w}$ re ausgeführten sehr ausführlichen Arbeit ubber den gleichen Gegenstand. Eine genaue Einsicht der Originalarbeit ${ }^{1}$ zeigte mir, dafs $A$. $D$ e w è $w$ re bei der mikroskopischen Untersuchung von den gleichen Gesichtspunkten ausging und auch im Wesentlichen zu ähnlichen Resultaten gelangte, wie ich. Ich werde daher bei Aufzählung der von mir gefundenen Ergebnisse das Unwesentliche fortlassen und mich mis der Angabe des

1) Dewèwre, Rtcherches s. le cubèbe otc. Annales publ. p. la soc. royale des seiences med. et natur, de Bruxelles, T. 31894. 
Hauptä̈chlichen begnügen. Obgleich dasselbe mit den von $D$ e w è we gefundenen Ergeb nissen im Grolsen und Ganzen tbereinstimmt, so glaube ich die Resultate meiner Untersuchungen in ihren hauptsächlichsten Zügen auch noch mitteilen zu sollen, einmal, da ich in einigen Punkten zu anderen Resultaten gelangte als Dewèwre und da ich weiter glaube, dal's auch die Mitteilung derjenigen Thatsachen, die mit den von $\mathrm{D} \theta \mathrm{w}$ è $\mathrm{w} \boldsymbol{r} \theta$ gefundenen ubereinstimmen, nicht überflüssig erscheint, weil sie immerhin eine Bestätigung bringen

\section{B. Pharmakognostischer Teil.}

Beschreibung der echtien Cubebe.

Bevor ich zur Beschreibung der falschen Cubeben übergehe, erscheint es mir notwendig, an dieser Stelle eine Besprechung des Baues der echten Cubebenfrucht einzuschalten, da die Angaben über denselben teilweise nicht korrekt, teilweise nicht erschöpfend sind.

Die Frucht entwickelt sich aus dem Fruchtknoten und dem orthotropen Ovulum; dieses, welches den Samen liefert, hängt auch in der reifon Frucht nur am Grunde mit dem Pericarp zusammen, die Samenschale verwächst also nicht mit letzterem, wie es beim Pfeffer und bei zahlreichen anderen Piperaceenfrüchten, die später als Verfälschungen der Cubeben zu betrachten sein werden, der Fall ist. Es kann daher beim Durchschneiden der Frucht der. Samen mit Leichtigkeit losgelöst werden.

Betrachten wir zmeächst den Querschnitt durch das Pericarp (Fig. II), so sehen wir als äulserste Schicht die von einer Cuticula bedeckte Epidermis (ep.) Die stark verdickten Aussenwände der Epidermiszellen zeigen auf der Innenwand Eiakerbungen. Die Zollen selbst sind langgestreckt, polyëdrisch und mit einem braunen Farbstoff an. gefullt, welcher der Droge die bekannte Farbe verleiht. In ihnen erkennt man bei aufmerksamer Beobachtung und genügend starker Vergrölserung vereinzelt rhombische Oxalatkrystalle. In gröfseren Mengel, wie sie $D \theta w$ è $w$ r gesehen zu haben angiebt, (derselbe fand die Zellen damit vollgestopft), habe ich dieselben auch bei Durchmusterung einer grofsen Anzahl Schnitte niemals beobachten können.

Es folgt nun nicht wie meistens angegeben wird, die äufsero Steinzellenschicht (a. st.) unmittelbar unter der Epidermis, sondern dieselbe wird von letzterer oft ganz durch eine aus $1-3$ Zellagen bestehende Schicht, welche nicht tarbstoffhaltig ist, getrennt. An diese Zellen lehnt sich die bypoepidermale, hie und da von parenchy-

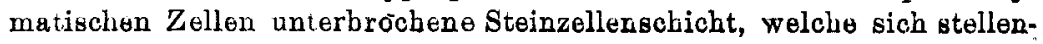
weise durch Aneinanderlagerung von 2-3 Sklereiden verstärkt. Die" Gestalt der Steinzellen ist eine mannigfaltige, vorwiegend polyedrische. 
Sie sind verhältnismälsig stark verdickt und mit zablreichen Tüpfeln versehen.

An diese Sklereidenschicht legt sich unmittelbar an die räumlich weitaus bedeutendete, aus Parenchymzellan gebildete Schicht des Pericarps. Sie lälst sich deutlich in eine breite, änfsere (ap.), bis zu den Gefäfsbundeln reichende und in eine schmälere, innere (ip.) gliedern. Erstere besteht aus grolsen dünnwandigen, Stärke und fettes Oel enthaltenden Zellen. Die zahlroichen kleinen Fetttröpfchen färben sich beim Behandeln mit konz. Schwefelsäure vorübergehend schon blau. Aufserdem finden sich eingestreut in diese Schicht die zahlreichen, aetherisches Oel führenden Sekretzellen $(0 \theta)$, in welchen man häufig kleine, aus Cubebinkrystallen bestehende Nadeln erkennen kann. Die Wandungen dieser Zellen sind verkork t.

Es fiel mir auf, dafs die Stärkekörner des Pericarps zuweilen nicht als differenzierte Körner, sondern mehr oder weniger als formlose Masse: Stärkekleister, bei der Behandlung mit Jod zu erkennen sind. Es wird dieses darauf zuräckzufubren sein, dals die Eingeborenen die Früchte mit heifsem Wasser abbrühen, die Błharddlung aber nur so kurze Zeit ausdehnen, dafs wohl das Amylum des Pericarps, nicht aber dasjenige des Perisperms davon beeinflufst wird. Von diesem Abbrühen berichtet schon G a rcia a b Orta (16. Jahrh.), es sollte den $Z_{w e c k}$ haben, die anderweite Kultur der Cubebe unmöglich zu machen.

Die ion re, schmälere Schicht wird von der eben beschriebenen durch die Gefälsbündel (g) getrennt. Dieselben bestehen aus wenigen Spiral- oder Ringgefälsen und kleinem Phloëm, vor dasselbe sind einige verdickte, poröse Fasern gelagert. Es unterscheidet sich diese Schicht wesentlich von der zuerst erwähnten. Ihre Zellen, morphologisch völlig identisch rait den dünnwandigen Parenchymzellen der breiten Schicht, sind in tangentialer Richtung molr zusammeu gedrückt, die Wandungen sind stärker und sie sind meistens mit Tüpfeln reichlich versehen. Bei Behandlung mit Phloroglucia und Salzsäure nehmen sie tine intensiv rote Farbe an: sie sind also verholzt. Das Auftreten der Sekretzellen ist in dieser Schicht weit weniger häufig, meistens enthält sie auch kin oder doch nur weniger Amylum. Ich will hier bemorken, dafs ich auf die $G$ :genwart von Amylum keinen Wert lege, ich habe Cubsben derselben Abstamming unter Händen gehabt, von denen marıche Früchte gar keine Stärkereaktion im Pericarp erkennen liefuen, andere gaben bald stärkere, bald schwächere in beiden Schichten. Es ist dieses ja auch leicht erklärlich, da der Gehalt an Amylum je nach dem Reifezustande ein wechselnder ist, wenn man bedenkt, dais Cubeben derselben Sendung nicht slle gleich reif, bezw. gleich unreif zu sein brauchen. Es ist 
anzune hmen, dafs vollständig reife Cubeben in der Fruchtschale gar kein Amylum enthalten.

Wir gelangen nunmehr zu der inneren Sklereidenschicht (i. st). Im Grolseli und Ganzen wird dieselbe von einer Lage dicht aneinander liegender grolser Steinzellen gebildet, stellenweise wird sie aber verstärkt durch eine, seltener durch zwei darüber liegender kleinerer Sklereiden. Die gröfseren Steinzellen sind radial gestreckt, reichlich mit Tüpfeln versehen und ihre Wandungen stark verdickt. Innerhall ditser inneren Steinscbale folgt schliefslich noch eine Schicht zu. sammengeprefster Zellen, die bisher vielfach übersehen zu sein scheint. Nur an besonders gănstigen Schnitten lälst sich erkennen, dafs sie aus mehreren Zelllagen und zwar sehr wahrscheinlich, wie beim Pfeffer, aus zweien solcher besteht.

Hiermit schlielst die Betrachtung des Baues des Pericarps. Der Querschnitt durch den Samen zeigt zunächst als äufserste Schichten die aus dem Tegumente hervorgegangene Samenschale. Dieselbe läfst zwei Schichten erkennen, eine äufsere mit ziemlich stark verdickten Wanden und einem braunen Inhalte, welcher mit Eisenchlorid die Gerbstoffreaktion giebt und eine innere aus ganz flachen Zellen bestehende, die häufig den Eindruck der stark verdickten Aussenmembran der Epidermiszellen des Perisperms macht und bisher auch als solche aufgetafst wurde. Die einzelnen Zellen bejder Schichten sind mehr oder weniger rechteckig gestreckt und zwar bei der unteren in umgekehrter Richtung wie bei der oberen, so dafs sich die Zellen bald mehr, bald weniger kreuzweise decken.

Der Samenkern besteht aus dem Perisperm, dem Endosperm und dem Embryo. Letzterer ist, wie bei den Piperaceen im Allgemeinen nur klein, er liegt im Endosperm eingebettet, naho der Spitze des Samens. Das Endosperm, das ebenso wie der Embryo in der trockenen Droge meist ganz zur Unkenntlichkeit verschrumpft ist, enthält, wenn es einigermalsen erbalten ist, fettes Oel und Aleuron. Der weitaus gröfste Teil des Samenkerns wird von dem Perisperm ausgefüllt. Dasselbe besteht au der Peripherie aus kleinen, vieleckigen Zellen, die nach der Mitte zu allmählich gröfser werden und radiale Streckung zeigen. Ein Teil der Zellen ist mit ätherischem Oel angefüllt und enthält, ebenso wie die Oelzellen des Pericarps. denen sie überhaupt analog sind, Cubebin. Der grölste Teil der Zellen enthält Stärke und Protoplasma. Erstere tritt in zwei verschiedenen Formen auf, sowohl als hochzusammengesetzte, wie auch in Gestalt einzelner kleiner Körner. Die hochzusammengesetzten Körner sind $37,5-53,5 \mu$ breit und $42,8-85,6 \mu$ lang, während die kleineren die Grösse von 1,8 bis $14,3 \mu$ haben. Letztere lassen nicht erkennen, dafs sie durch Zerfall von hochzusammengesetzten stärkekörnern entstanden sind. Es mufs besondere hervorgehoben werden, dafs die Zellen mit Einzelkörnern 
nicht etwa nur in der Nähe der Peripherie des Perisperms vorkommen, sondern dafs in manchen Frächten die Stärke durchweg in Einzelkörnern vorhanden ist.

Aufser Stärke und Protoplasma können in diesen Zellen noch andere kleine Kügelchen erkannt werden, teils einzeln, teils aneinander gelagert, welch $\theta$ sich mit Jod nicht blau, sondern gelb färben. Durch einfaches Färben der Zellen mit Jod können dieselben nur schwer erkannt werden, da die starke Blaufärbung der Stärke die Farbe der in geringerer Menge als diese vorhandenen Körner fast vollständig verdeckt. Es empfiehlt sich daher, das Amylum durch Behandeln mit Salzsäure oder Natronlauge zu entfernen, bevor man Jod hinzufügt. Man könnte geneigt sein, diese Körner für Aleuron zu halten, allein die genauere Untersuchung zeigt das Unhaltbare dieser Annahme. Sie lösen sich nicht in Wasser, weder in Salzsäure noch in Natronlauge (worin sie etwas aufquellen), und auch nicht in Natriumphosphatlösung. Durch Eisenchlorid werden sie nicht gefärbt. Da diese Körner durch Jod gelb, durch Millon's Reagens rot und durch Salzeäure-Carmin rötlich gefärbt werden, also die spezifischen Reaktionen des Proteîns zeigen, so müssen dieselben als Bestandteile des Plasma's angesehen werden. Es dürften wohl diese Körner dasjenige vorstellen, was Tschirch unter dem Namen "Füllstärks" beschreibt.

Es ist mehrfach behauptet, nur das Perisperm, nicht aber das Pericarp enthalte Cubebin. Ich halte diese Behauptung für unrichtig. Der Nachweis des Cubebins lälst sich mikrochemisch mit Leichtigkeit und grolser Schärfe führen. Cubebin giebt, wie schon mebrfach erwähnt, mit konz. Schwefelsäure eine intensiv purpurviolette Färbung, die derjenige, welcher sie einmal gesehen hat, gar nicht mit anderen Färbungen verwechseln kann; versetzt, man Cubebin mit etwas Ammoniummolybdat und fügt konz. Schwefelsäure hinzu, so geht die zuerst auftretende Purpurfärbung bald in ein intensives Blau über, ferner nimmt Cubebin mit Phosphorsäureanhydrid nach einiger Zeit eine rotviolette Fürbung an. Diese drei sehr scharfen Reaktionen eignen sich ganz besonders zum mikrochemischen Nachweis. Die Ausführung geschieht, indem man die dünnen Schnitte unter dem Deckglä日chen mit einem Tropfen Schwefelsäure behandelt, oder indem man dieselben zunächst mit einer konz. Lösung von Ammoniummolybdat auf dem Objektträger befeuchtet, die Flüssigkeit nach einigen Minuten durch Absaugen mittels Filtrierpapier möglichst entfernt und dann ein wenig konz. Schwefelsäure hinzufliefsen läfst, oder indem man die Schnitte auf dem Objektträger mit etwas $P$ hosphorsäureanbydrid bedeckt, einige Zeit an freier Luft liegen lälst, bis dasselbe etwas Feuchtigkeit an. gezogen hat. Bei allen drei Reaktionen sind die bezüglichen charakteristischen Färbungen des Cubebins sehr deutlich wahrzunehmen und zwar nicht nur in den aus dem Perisperm hergestellten Schnitten, 
sondern auch und zwar mit gleicher Schärfe, in solchen aus dem Poricarp.

Das Cubebin ist in der lebenden Pflanze in den Sekretzellen enthalten; bei Ausfübrung der genannten Reaktionen in oben angege. bener Weise, beobachtet man aber nicht nur in diesen Zellen, sondern oft im ganzen Gewebe das Auftreten der charakteristischen Färbungen. Es muls also angenommen werden, dafs das Oubobin nach dem Absterben dieser Zellen aus denselben in das umliegende Gewebe übergeht, trotzdem die Wandungen der Sekretbehälter verkorkt erscheinen, denn die Möglichkeit, dafs bei Anfertigung der Schnitte das ätherische Oel mit dem Cubebin über die ganze Ausdehnung derselben verbreitet werden könnte, kann wohl nicht als alleiniger Gruad für das Auftreten der Färbungen im ganzen Gewebe angesehen werden, da die Färbung ganz gleichmässig ist und bei allen Schnitten auftritt.

Ioh will hierbei bemerken, dafs das eben Gesagte, mutatis mutandis, auch auf die Pfefferfrucht anzuwenden ist. Auch von dieser wird behauptet, dal' Piperin nicht im Pericarp, sondern ausschlielislich im Perisperm vorkomme ${ }^{1}$ ). Es lä ('st sich hier die Reaktion mit Ammoniummolybdat und Schwefelsäure zur Bestätigung der Anwesenheit des Pipering im Perisperm a n $\mathrm{dm}$ Pericarp verwenden. (Auch Tschirch und $\mathrm{Oester} \mathrm{le}^{2}$ ) scheinen die Gegenwart von Piperin im Perisperm und im Pericarp anzunehmen.) Das von Herlant1) 2um Nachweis des Piperins gebrauchte Chloralbydrat, durch welches bei Gegenwart des ersteren eine gelbe Färbung entstehen soll, scheint mir nicht besonders glicklich gewä!lt und zwar aus dem einfachen Grunde, weil die Sekretzellen von Piper nigrum unter dem Mikroskope, auch schon ohne irgend welche Behandlung mit Chloralhydrat, intensiv gelb gefärbt erscheinen.

Es sei hier bemerkt, dafs mikrochemisch die erwähnte Resktion mit Piperin und Cubebin in nahezu gleicher Weise verläuft. Allerdings ist die zuerst auftretende Rotfärbung bei beiden verschieden doch ist hierauf kein grofser Wert zu legen, da dieselbe zu bald einer bei beiden Körpern in gleicher Weise auftretenden blauen Farbe Platz macht. Die bei langer Einwirkung der Reagention auftretenden Farbentöne lassen sich ebenfalls nicht gut zur Unterscheidung von Cubebin und Piperin heranziehen, da die energische Wirkung der konz. Schwefelsäure auf das Pflanzengewebe dieselben störend beeinflufst. Der Wert der Reaktion wird deshalb aber durchaus nicht herabgemindert, da bis jetzt noch kein Fall bokannt ist, dals die genannten beiden Substanzen in einer and derselben Pflanze vorkommen.

1) Herlant, Analyse du poivre de Clusii. Bullet. acad. de méde. cine de Belg. 1894.

2) Tschirch und Oesterln. Anatom. Atlas 1894. 
Makrochemisch läfst sich aber Cubebin, Piperin und auch das ven mir aufgefundene und später zu bes chreibende Pseudocubebin mit Sicherheit durch diese Reaktion unterscheiden und zwar sowohl durch die anfängliche Färbung, welche bei Cubebin purpurviolett, bei Piperin blutrot und bei Pseudocubebin gelbbraun ist, als auch durch die Färbung, welche nach der allen drei Körpern gemeinsamen Blaufärbung eintritt. Diese ist bei erstgenanntem Körper ein reines, tiefes, bleibendes Blau, bei dem zweiten ist sie bläulichgrün und bei dem dritten rötlichblau. Die Reaktion verläuft mit um so grölserer Schärfe, je weniger Substanz and je geringere Menge Scbwefelsäure in Anwendung kommt.

Ich möchte hier kurz das Ergebnis einer mikrochemischen Untersuchung über die Verteilung des Cubebins in der Pflanze einschaltan. Dieselbe erstreckte sich sowohl auf männliche als weibliche Exemplare von Piper Cubeba, var. Katoentjar. Es zeigte sich, dafs das Cubebin seiuen Hauptsitz in den Früchten selbst hat, ausserdem findet aich daseelbe, jedoch in weit geringerer Menge, in den Fruchtspindeln, während Wurzel, Stamm, Blattstiel and Lamina sich als vollständig frei von Cubebin erwiesen. Die in den Markstrahlen der Wurzel besonders zahlreich vorhandenen Sekretbehälter geber mit konz. Schwefelsäure eine deutliche Grünfärbung.

Beschreibung der Verfälschungen und Substitutionen.

Die Verfälschungen der Cubeben lassen sich zunächst in drei Hauptgruppen einteilen :

I. Piperaceenfrüchte mit stielartigem Fortsatz des Pericarps.

II. Sitzende Piperaceenfrüuchte.

III. Früchte aus anderen Familien.

Während die beiden letzteren ohne weiteres als Verfälschung zu erkennen sind, bringt der, der echten Cubebe oftmals sehr ähnelnde Bau der ersten Gruppe Schwierigkeiten mit sich. Es lälst sich diese Gruppe in vier Unterabteilungen zerlegen, und ich stütze mich bei Aufstellung derselben auf das auch dem ungeübteren Mikroskopiker am meisten in die Augen fallende Merkmal, auf die Verteilung der Steinzellen im Pericarp.

Ich unterscheide hiernach :

1. Früchte mit innerer und äufserer Sklerenchymschicht aufserdem aber vereinzelte oder gruppenweise Steinzellen im Parenchym (vergl. Fig. 1.) 
2. Solche mit innerer und äufserer Sklerenchymschicht, also vom Bau der echten Cubobe (vergl. Fig. II).

3. Aeufsere Steinzellenschicht vorhanden, meist sehr schwach entwickelt, innere aber gänzlich fehlend (vergl. Fig. III).

4. Früchte ohne Sklerose (vergl. Fig. IV).

\section{Piperaceenfrüchte mit stielartigem Fortsatz des Pericarps.}

1. Aeussere nnd innere Steinzellenschicht vorhand en, ansserdem zerstrente Sklerose im Parenchym des Pericarps (Fig. 1.)

$$
\text { Piper ribesioides Wallich. }
$$

Breite $7 \mathrm{~mm}$, Länge $7 \mathrm{~mm}$, Stiel $7-8 \mathrm{~mm}$. (Ich bemerke, dals sich diese Angaben überall auf die in Wasser vollständig aufgeweichten Früchte beziehen und zwar ist stets das Mittel aus einer Anzahl Messungen angegeben.)

Die Früchte sind etwas grölser als die der echten Cubebe, der Stiel nur wenig länger als die Frucht, ihre Farbe durchläuft so ziemlich alle Schattirungen von hellbraun bis schwarzbraun, der Same ist nur am Grunde mit dem Pericarp verwachsen. Auf der Grenze der äufseren und inneren Parenchymschicht des Pericarps finden sich grolse halbmondförmige, nach der inneren Sklerenchymschicht zu gebogene Höhlungen (h), deren äufsere Wände mit kleinen, meist getrennt liegenden Sklereiden besetzt sind. Diese Höhlungen sind so grols, dafs sie immer die Zwischenräume zwischen je zwei Gefärsbündeln ausfüllen, so dafs die innere (ip) und äufsere (ap) Parenchymschicht nur durch die Gefälsbündel (g) und deren nähere Umgebung zusammenhängen. $\mathrm{D} \theta \mathrm{w}$ \& $w \mathrm{r}{ }^{1}$ ) hält diese Höhlungen für lygigen entstanden. Wenn auch eine Entscheidung über ihre Entstehung nur auf Grund entwickelungsgeschichtlicher Untersuchungen möglich ist, so stehe ich doch nicht an, sie für schizogen entstanden zu halten. Sie dürften ihre Entstehung vielleicht Gewebespannungen, die durch stärkeres Wachstum der

1) Dewèwre. Recherches s. le cubèbe etc. annales, publ. p. la soc. royale des sciences med. et natur. de Bruxelles T. 3, 1894. 